\title{
Is there inanimate memory?
}

It is too easy to forget that pre-Victorian physics ascribed memory to lumps of iron. Now this still surprising phenomenon has a mathematical basis to call its own.

EVERYBODY knows what is meant by hysteresis, chiefly from what school physics teachers have had to say. Take a piece of magnetic material such as iron, put it in a magnetic field whose strength can be varied and, starting with unmagnetized iron, let the strength of the magnetic field increase steadily. To begin with, the magnetization of the iron increases less quickly than the field; anthropomorphically, the iron hankers after its unmagnetized condition. But then the rate of increase becomes greater; magnetization grows on what the economists call an Sshaped curve, the kind of thing that might be represented by a factor such as $[1-$ $\exp (-x)]$

The surprise is that the same happens on the way down. Decreasing the external magnetic field gives the impression that the lump of iron hankers after its magnetized condition. Plotting the values for magnetization and external magnetic field on a piece of graph paper, there is a gap between the two S-shaped curves. Many young people are asked to record the difference, but are rarely asked to think what it means. But these days, when school administrators are so concerned with technology, young people may be expected to know that the area of the hysteresis curve is a measure of the energy loss of a transformer using iron as a magnetic inductor.

The more interesting question is that of the physics of the process. How is it that a lump of iron can remember its past history in such a reproducible fashion that its hysteresis curve can be counted as a function of its constitution? To be sure, the area within the hysteresis curve is a function of the frequency with which the cycle is traversed (the faster, the greater the area), which implies that lumps of iron have not merely a memory, but a time-dependent capacity to forget. I.D. Mayergoyz from the University of Maryland has now provided a framework in which to ask how such odd behaviour may arise (Phys. Rev. Lett. 56,1518; 1986).

Mayergoyz's starting point is in 1935, and the work of the German physicist Preisach who is said (a little disdainfully) to have constructed a model of hysteresis on "some hypotheses concerning the physical mechanisms of magnetization. Abstractly, of course, magnetization followed by demagnetization must entail some kind of energy expenditure. A magnetized state is ordered, and so has

\section{lesser entropy than that from which it was} formed. Simple thermodynamics proclaims that achieving such a state in a finite time requires that some work should be done (which will eventually appear as heat). But that is no more illuminating about the underlying mechanism than the calculations economists make to show that a trade surplus (deficit) must always be balanced by an export (import) of capital, whence (in physics) concepts such as magnetostrictive friction, fashionable enough in the 1930s.

That is why the mathematical framework of the discussion may be helpful in its own right. Mayergoyz borrows heavily from the Soviet mathematician Kasnoselski, whom he describes as having distilled from earlier work a way of describing hysteresis. The problem is this. A hysteresis curve is a function in some appropriate space. An input, in the simplest case the changing strength of the external field as a function of time, yields an output characteristic of itself. If the speed of the transition from the unmagnetized to the magnetized condition is immaterial, or is ignored, there remains a problem in that the shape is a function of the initial and final values of the external field. More generally, at any point of the planar graph in which magnetization is represented as a function of external field, there is an infinity of curves that may represent the future behaviour of a lump of iron, each one dependent on a particular past history.

What emerges from this argument is a description very similar to what the statisticians would call a Markov system. The future behaviour (magnetization) of a lump of iron may be determined from a knowledge of its state at some instant and from a knowledge of the magnetic field then and at subsequent times. How could it be otherwise, when causality applies? So there must be something about the initial state that embodies the memory of recent past history. In dynamical systems, it may be the rate at which the system is changing on a microscopic scale (the velocity field); in the relatively static context of magnetization, the pattern of magnetization is the clue to the past.

The important part of Mayergoyz's result is his demonstration that it should be possible to calculate the properties of the operator that transforms the input function of the system (the external magnetic field) by means of a simple series of experiments in which the output (magnetization) is recorded. The demonstration is mathematical, and in a novel way, but the result is to suggest what the history of mathematical physics has consistently shown to be significant, the forging of relationships between functions of one kind and functions of another. The fact that, in the simplest case of hysteresis, that of magnetism, the results must accord with the thermodynamics of entropy and the explanation may lie in microscopic physics is, for the time being, irrelevant.

One practical implication of the argument stands out. Although a system showing hysteresis will behave in a particular way determined by its starting condition and the external influences upon it, there are general rules that determine the behaviour of systems exposed to extreme conditions that appear to be unaffected by the details of the case. This is mirrored, in Mayergoyz's treatment, by the way in which the local microscopic conditions (say, local magnetic domains), which are the local repositories of memories of the past, will be smeared out or extinguished by extreme conditions.

What may this mean for the concept of memory? The simple and oversimple answer is that there can be no connection between inanimate physical systems such as lumps of iron and biological systems, some even blessed with what is called consciousness, in which memory must be an infinitely more subtle business. But can that in reality be the case? Nobody seriously suggests that human beings, who can remember the past, are on that account exceptions to causality.

On the contrary, it is simplest to suppose that the state of the nervous system at any instant somehow mirrors past experience in such a way as to be able to regenerate images of the past that people call memories. What Mayergoyz has done is merely to show that such a model can represent what happens with inorganic materials that appear to have a memory of the past. That is not surprising in itself. The fun will come if it turns out that the equations explain some of the properties of memory as people know it. And if the physical analogy of magnetization shows that exceptional local patterns tend to be smeared out, what can explain in the human case the enhancement of especially vivid memories? It will be interesting if the tricks that people play on themselves can be derived from theorems of some kind. John Maddox 